\title{
Battlefield Analgesia - A Basic Approach
}

Flt Lt G Hocking

$\mathrm{MB}, \mathrm{ChB}, \mathrm{DA}(\mathrm{UK})$, RAF

Senior House Officer

\section{Lt Col WF de Mello}

FRCA, RAMC

Senior Lecturer in Anaesthetics (Army)

Department of Anaesthesia, Royal Hospital Haslar, Gosport, Hants PO12 2AA

SUMMARY: There is a requirement for a basic approach to the provision of analgesia on the battlefield using the morphine autojet. Assuming there is cardiorespiratory stability and no contraindications to opioid therapy, morphine can be administered intramuscularly to a serviceman in pain on a 2 hourly basis provided that there are no adverse effects on the respiratory rate and degree of sedation. This proven simple approach in civilian practice has the potential to be incorporated into Battlefield Advanced Trauma Life Support (BATLS) and Combat Training Regimens (CTR).

\section{Introduction}

The provision of appropriate analgesia to patients in pain has obvious benefits, yet it remains a subject that is poorly covered in medical and nursing education. In 1990, the Royal College of Surgeons and the Royal College of Anaesthetists published a joint document entitled "Pain after surgery" (1) which concluded that the treatment of pain after surgery in British hospitals had been inadequate and not advanced for many years. An audit of recovery room complications at the Cambridge Military Hospital, Aldershot, revealed that pain was the commonest complication and occurred in $32 \%$ of all patients (2). The traditional method of postoperative analgesia is intramuscular morphine which remains the gold standard despite its limitations and other advances like patient controlled analgesia, epidurals etc. Gould et al (3) improved the quality of intramuscular analgesia by using a simple protocol that allowed morphine to be administered hourly if necessary. Our own experiences and those of others (4) have confirmed the efficacy of this simple approach. Peacetime clinical experience obviously has to be adapted for the battlefield. We provide a basic approach to battlefield analgesia that can be used by the serviceman, either for self or buddy-buddy administration (Table 1).

\section{Discussion}

To develop a suitable protocol for the provision of analgesia on the battlefield we have taken into account the following.

1. Medical factors:

Servicemen are assumed to be ASA 1 or 2 (5) prior to injury (i.e. physically fit) with no known drug allergies, on no concurrent therapy and to have a full stomach.

\section{Logistic factors:}

The only drugs carried by the serviceman would be the $10 \mathrm{mg}$ morphine autojet (6) and the Combo pens for
Table 1

Basic Approach to Battlefield Analgesia

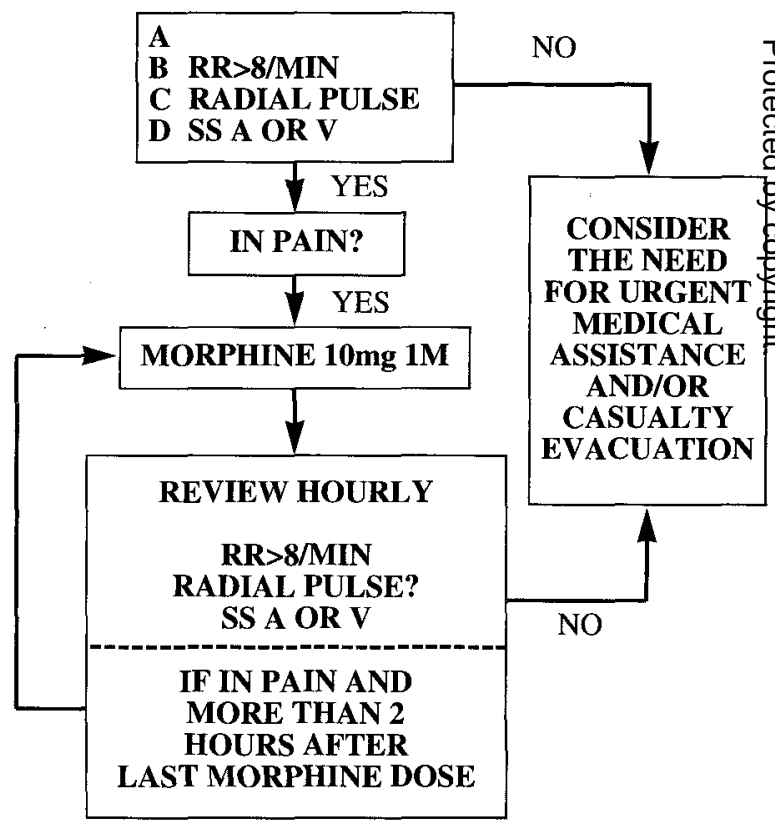

treatment of chemical poisoning. The morphine autojet is issued to servicemen with the same precautions as live ammunition with the understanding that the drug would be administered for pain relief where medical expertise and assistance was limited or not immediately available. Besides analgesia, morphine administration would also facilitate the quality of casualty evacuation of the injured serviceman.

3. Pharmacological factors:

The analgesic of choice must be familiar to the user, 
effective, easy to use, have minimal side effects and may be reversed by a specific antidote. We have selected morphine based on its pharmacological and pharmaceutical profile. We have chosen the intramuscular route into the vastus lateralis or deltoid muscle. Blood levels of morphine need to reach a minimum effective analgesic concentration (MEAC) for each individual patient before any pain relief is obtained. MEAC can vary some four to five fold between patients. Therefore the only way to obtain good analgesia is to titrate the dose of opioid for each patient. This titration is obtained by selecting an appropriate initial dose and /or dose interval. In addition the effectiveness of the analgesia and side effects of therapy must be monitored (i.e. degree of sedation, effect on respiratory rate, nausea, vomiting etc.). As the autojet has $10 \mathrm{mg}$ of morphine, the only alternative method of titration available on the battlefield is changing the dosing interval.

Traditionally the dose of morphine was based on body weight (7), when in fact there is no correlation between patient weight and opioid requirement. The best clinical predictor of opioid dose is in fact patient age (8). The average 24 hour morphine requirement for patients over 20 years is $100 \mathrm{mg}$ minus the age in years. Assuming frontline serviceman are aged between 18 and 40 and taking 24 years as an illustration, the morphine requirements over 24 hours is $100-24=76 \mathrm{mg}$. If this is divided into 8 aliquots this gives a $9 \mathrm{mg}$ dose at 3 hourly intervals. But in order to improve the quality of the analgesia we have reduced the dose interval to 2 hours as required, provided there is no deleterious effect on the respiratory rate or on the degree of sedation. We have defined deleterious as a respiratory rate of 8 or less per minute and/or an unacceptable degree of sedation as $\mathbf{P}$ or $U$ of the AVPU score in Advanced Trauma Life Support teaching where A is Alert, $\mathrm{V}$ is Verbally responsive, $\mathrm{P}$ is responsive to Painful stimuli and $U$ is Unresponsive (9).

4. Built in safety

The analgesic algorithm is only commenced after cardiorespiratory stability is obtained on the Primary Survey or subsequent Secondary Survey, and that vital signs are monitored regularly. We feel that recommendations for repeated observations, seeking medical assistance and considering casualty evacuation are added safety features. The simplicity of the algorithm is "user friendly" to the potential user/administrator.

5. Clinical experience

We have tested the efficacy of the protocol in peacetime practice in military hospitals and this has been confirmed by civilian colleagues ( $G$ Bowler, personal communi-cation 1996).

We suggest that a protocol like this could be adopted as part of BATLS and CTR in providing a basic approach to battlefield analgesia.

\section{Acknowledgement}

We are grateful to Colonel P Roberts late RAMC for his support and encouragement in the preparation of this project.

\section{Addendum}

Further refinements to this basic approach and other. methods of analgesia that could be provided by general duties medical officers and anaesthetists will be covered in a future publication.

\section{REFERENCES}

1. Royal College of Surgeons of England, College of Anaesthetists. Commission on the provision of surgical services. Report of the working party on pain after surgery. 1990; London: Royal College of Surgeons.

2. DEMELLO WF. Overview of complications. In: Gran

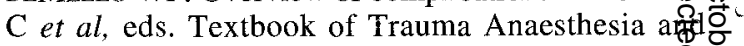
Critical care. St Louis: Mosby Year Book, 1993.

3. Gould TH, Crosby DL, Harmer M, et al. Policy far controlling pain after surgery: effect of sequentigl changes in management. Br Med $J$ 1992; 305: $118 \overline{8}$ 93.

4. Macintyre PE, Ready LB. Acute Pain Management. A practical guide. London: WB Saunders, 1996.

5. American Society of Anaesthesiologists. New classification of physical status. Anaesthesiology 1963; 24:

6. Further information on the morphine autojet available from STI International Ltd, ROCHESTER, Kent, ME2 8DP, England.

7. LASAgna L, Beecher HK. The optimal dose of morphine. J Am Med Assoc 1954; 156: 230-34.

8. MACINTYRE PE, JARVIS DA. Age is the best predictor of postoperative morphine requirements. Pain 1996; 64: $357-364$.

9. American Trauma life Support Manual for Physicians. American College of Surgeons Committee on Trauma. Chicago: 1993. 